\title{
Genome-wide (ChIP-seq) identification of target genes regulated by BdbZIP10 during paraquat-induced oxidative stress
}

\author{
Ruth C. Martin ${ }^{1^{*}}$, Kelly Vining ${ }^{2}$ and James E. Dombrowski ${ }^{1}$
}

\begin{abstract}
Background: bZIP transcription factors play a significant role in many aspects of plant growth and development and also play critical regulatory roles during plant responses to various stresses. Overexpression of the Brachypodium bZIP10 (Bradi1g30140) transcription factor conferred enhanced oxidative stress tolerance and increased viability when plants or cells were exposed to the herbicide paraquat. To gain a better understanding of genes involved in bZIP10 conferred oxidative stress tolerance, chromatin immunoprecipitation followed by high throughput sequencing (ChIP-Seq) was performed on BdbZIP10 overexpressing plants in the presence of oxidative stress.

Results: We identified a transcription factor binding motif, TGDCGACA, different from most known bZIP TF motifs but with strong homology to the Arabidopsis zinc deficiency response element. Analysis of the immunoprecipitated sequences revealed an enrichment of gene ontology groups with metal ion transmembrane transporter, transferase, catalytic and binding activities. Functional categories including kinases and phosphotransferases, cation/ion transmembrane transporters, transferases (phosphorus-containing and glycosyl groups), and some nucleoside/ nucleotide binding activities were also enriched.
\end{abstract}

Conclusions: Brachypodium bZIP10 is involved in zinc homeostasis, as it relates to oxidative stress.

Keywords: bZIP transcription factors, Oxidative stress, Zinc, Chromatin immunoprecipitation (ChIP), Brachypodium

\section{Background}

Transcription factors (TFs) are integral for the regulation of gene expression during the normal growth and development of all organisms and for facilitating responses to environmental and physiological stresses. As organisms become more complex, different families of TFs may increase in number and diversify in function [1]. This is evident in plant TFs such as the Dof (DNAbinding one finger) gene family [2], MADs-Box gene family [3] and the bZIP gene family in plants [4]. These bZIP TF families contain groups with members that control networks of genes that may be distinct from those controlled by their ancestral TF gene [5].

Within the plant kingdom, bZIP TFs are proposed to have evolved from four founder genes that were involved in oxidative stress and the unfolded protein response [5].

\footnotetext{
* Correspondence: Ruth.Martin@ars.usda.gov

1 USDA ARS National Forage Seed and Cereal Research Unit, 3450 SW

Campus Way, Corvallis, OR 97330, USA

Full list of author information is available at the end of the article
}

As plants have evolved, the number of bZIP TFs has increased, and their roles have expanded to include many aspects of plant growth including embryo development, germination, shoot development, flowering, and seed development [6]. The bZIP TFs also play critical regulatory roles during plant responses to various stresses including cold, drought, salt, heat, and submergence $[5,7]$.

The genomes of many plant species have been mined for genes encoding bZIP proteins, and the number of these proteins varies widely in different species, from 75 in Arabidopsis [8], 55 in Vitis vinifera L. [9], 131 in Glycine max (L.) Merr. [10], 272 in Gossypium raimondii Ulbr., and 95 in Brachypodium distachyon [11]. The four progenitor groups of bZIP proteins have evolved and are subdivided into 13 groups (A-L and S) based on their bZIP domains, conserved motifs, and the presence of introns in the coding region for the conserved basic motif of many angiosperms [5]. While the total number of bZIP proteins varies in different species, all plants have representatives within each of the 13 groups. Within 
each group, the possible groups of orthologous members have diverged from the same ancestral gene and usually are associated with similar processes; for example within Group A, many of the TFs are involved in abscisic acid related processes, many Group $\mathrm{G}$ members are involved in light responses and photomorphogenesis, Group C and $S$ members tend to function in energy metabolism and oxidative stress responses, Groups D and E are involved in hormonal responses and plant development, and Groups $\mathrm{K}$ and B may be involved in the unfolded protein response [5]. Less is known about Groups F, J and L. Members of Group F-subgroup 1 are widely conserved across land plants [12], and two members of this subgroup, AtZIP19 and 23, have been characterized as zinc deficiency TFs [13]. More recently, the Atbzip19 single mutant was shown to be unable to adapt to zinc deficiency conditions, while the Atbzip23 single mutant was not severely affected under the same conditions [14]. Group F-subgroup 2 contains AtbZIP24, which is involved in abiotic stress responses [15], and is more variable across land plants [12].

Zinc deficiency was previously shown to induce oxidative stress related genes in wheat [16]. Furthermore, the BdbZIP10 (Bradi1g30140) TF, when overexpressed in Brachypodium, conferred enhanced oxidative stress resistance and increased viability when plants or cells were exposed to $\mathrm{N}, \mathrm{N}^{\prime}$-dimethyl-4,4'-bipyridinium dichloride (common name: paraquat) [17], an herbicide which induces oxidative stress in plants $[18,19]$. This BdbZIP10 TF is the homolog of Arabidopsis bZIP19 and Arabidopsis bZIP23 TFs, which have been characterized for their role in adaptation to zinc deficiency in Arabidopsis. Since overexpression of BdbZIP10 increases oxidative stress tolerance [17], we investigated which genes this TF interacts with during the oxidative stress tolerance response. Chromatin immunoprecipitation followed by high throughput sequencing (ChIP-Seq) is valuable for identifying DNA binding sites of transcription factors [20, 21]. Paraquat-treated Brachypodium overexpressing the BdbZIP10-GFP fusion gene was used for chromatin immunoprecipitation using a GFP antibody that recognizes the BdbZIP10-GFP fusion protein [17] in order to identify potential downstream targets of BdbZIP10 during response to oxidative stress.

\section{Methods}

\section{Brachypodium growing conditions and stress treatment}

The production and characterization of bZIP10-GFP over-expressing Brachypodium was previously described [17]. Briefly, cDNA was prepared from oxidativestressed leaf tissue of Brachypodium distachyon and was used as a template to amplify the bZIP10 gene (Bradilg30140), which was inserted into a modified pART vector [22] under the control of the ZmUbi1 promoter, with a C-terminal GFP tag. The construct was transformed into Agrobacterium strain AGL1 [23], which was used to transform Brachypodium distachyon. Plants transformed with this ZmUbi1::bZIP10-GFP construct developed normally compared to the wild type plant [17].

Seeds of Bd21-3 or bZIP10-GFP transformed Brachypodium distachyon [17] were planted in SB40 Sunshine Growing Mix (Sun Gro Horticulture Inc., Bellevue, WA) with Osmocote Plus 15-9-12 (The Scotts Company, Marysville, $\mathrm{OH}$ ) added at planting. After planting, the pots were placed at $4{ }^{\circ} \mathrm{C}$ with $8 \mathrm{~h}$ day length for $5 \mathrm{~d}$ to synchronize germination. Plants were then placed in a Conviron PGV36 growth chamber (Conviron, Winnipeg, Canada) set for $20 \mathrm{~h}$ light at $24{ }^{\circ} \mathrm{C}$ and $4 \mathrm{~h}$ dark at $18{ }^{\circ} \mathrm{C}$. Plants were grown for at least six weeks postgermination before leaf tissue was harvested for ChIP sequencing and expression experiments. Paraquat treatments were performed using a modified version of existing leaf toxicity protocols $[17,24,25]$. For paraquat leaf treatment, 7 - to $10-\mathrm{cm}$-long leaves were harvested, placed in fresh solutions of $160 \mu \mathrm{M}$ paraquat, vacuum infiltrated for $5 \mathrm{~min}$ with house vacuum, and placed on a gentle rocker under continuous light for $24 \mathrm{~h}$ at room temperature. For ChIP, paraquat-treated leaf tissue was rinsed briefly in sterile distilled water prior to formaldehyde cross-linking with $1 \%$ formaldehyde in phosphate buffered saline (PBS) under vacuum infiltration for $20 \mathrm{~min}$. Cross-linking was terminated by adding glycine (125 $\mathrm{mM}$ final concentration) directly to the samples and applying vacuum infiltration for an additional $10 \mathrm{~min}$. The tissue was rinsed, blotted dry, and then ground to a fine powder in liquid nitrogen.

\section{ChIP-sequencing and ChIP-qPCR}

ChIP-Seq service was performed by Zymo Research Corporation (Zymo Research Corp., Irvine, CA). Chromatin was prepared using the Zymo-Spin ${ }^{\mathrm{mm}}$ ChIP kit (Zymo Research Corp., Irvine, CA) following their modified protocol for isolation of plant chromatin. Sonication was performed at high power setting for 40 cycles (30 s on, $30 \mathrm{~s}$ off) using a Bioruptor Plus ${ }^{\text {Tx }}$ (Diagenode Inc., Denville, NJ), yielding a DNA fragment-size range of 200-700 bp. The ChIP assay was performed in triplicate $(N=3)$ using $12 \mu \mathrm{g}$ of chromatin and $10 \mu \mathrm{g}$ of anti-GFP ChIP Grade antibody (Abcam ab290, lot \#GR240324-1; www.abcam.com) to target the bZIP10-GFP protein. Anti-IgG (Millipore, PP64B; Lot 2,565,474) at a concentration of $10 \mu \mathrm{g}$ was included as a negative control. ChIP DNA was purified using the ChIP DNA Clean and Concentrator $^{\text {rm }}$ (Zymo Research Corp, Irvine, CA). ChIP-Seq libraries were prepared and sequenced on a HiSeq 1500 sequencer (Illumina, San Diego, CA). ChIP-Seq reads were aligned to the Brachypodium diastachycon reference genome (version 3.1) by Bowtie [26] with at most 2 
mismatches. Reads that appeared more than twice at the same position on the same strand were discarded to remove PCR duplication. BigWig files were generated from the alignment for visualization purposes [27]. MACS2 (Model-based Analysis of ChIP-Seq) [28] was used to identify peaks using a q-value cutoff of 0.05 .

In addition, ChIP-qPCR analysis was performed by Zymo Research Corporation. Briefly, 10 unique primer sets were designed to amplify a range of enriched regions identified in the GFP ChIP sequencing data. Anti-GFP ChIP and IgG negative control DNA enriched from two independent ChIP assays were amplified using ZymoTaq ${ }^{\mathrm{Tm}}$ qPCR Premix (Zymo Research Corp., Irvine, CA). ChIP DNA enrichment was determined as \% of input (i.e., the relative amount of immunoprecipitated DNA compared to $100 \%$ input DNA after qPCR analysis).

\section{Motif analysis}

To identify possible binding motifs of the bZIP10 transcription factor, the ChIP peak sequences were subjected to MEME (Multiple EM for Motif Elicitation)-ChIP [29]. The MEME-ChIP program uses two ab initio motif discovery algorithms: MEME [30], and DREME (Discriminative Regular Expression Motif Elicitation) [31], which uses regular expressions to search for short eukaryotic TF motifs that are missed by MEME.

\section{Gene function of BdbZIP10 TF target genes}

In order to determine putative functions of BdbZIP10 target genes, the set of 259 genes with ChIP-Seq peaks located in upstream promoter regions or potential downstream regulatory regions (2 kb upstream of transcription start site or $2 \mathrm{~kb}$ downstream of stop codon) or within annotated gene bodies were analyzed based on Gene Ontology (GO) categories using the AgriGO online Gene Set Enrichment Analysis (GSEA) tool [32, 33]. The default Fisher's Exact Test and Benjamini-Yekutieli multiple test correction methods [34] were employed to generate $p$-values for statistical significance and corresponding False Discovery Rate (FDR) values.

Expression analysis of several genes with ChIP-fold enrichment greater than ten was examined to determine their expression levels in the presence or absence of oxidative stress (paraquat treatment). Three BdbZIP10-GFP OE lines (from three independent transformation events) were compared to three WT plants undergoing the same treatment (water or water with paraquat) for this analysis.

\section{RNA extraction and quantification}

Three BdbZIP10-GFP OE lines (from independent transformation events) were compared to WT plants undergoing the same treatment for gene expression analysis. RNA extraction was performed with the Direct-zol ${ }^{\text {ma }}$ RNA MiniPrep System (Zymo Research Corp., Irvine,
CA). Briefly, $250 \mathrm{mg}$ of leaf pieces were ground in liquid nitrogen, transferred to Trizol, and then column purified, including the DNase I digestion step, following the manufacturer's instructions. Approximately $3 \mu \mathrm{g}$ of RNA was used for cDNA synthesis using the SuperScript ${ }^{\oplus}$ III First-Strand Synthesis System (Invitrogen) following the manufacturer's instructions.

Quantification of cDNA was performed using the BioRad Real Time PCR detection system and the Biorad iTaq $^{\text {Tn }}$ Universal SYBR Green Supermix following the manufacturer's instructions (Bio-Rad Laboratories, Hercules, CA). Primers were designed with QuantiPrime [35] using the website's default settings, and tested for linearity. Primer sequences used in this study are listed in Additional file 1. For RT-qPCR, BdUBC18 was used as a normalization housekeeping control gene [36] and the comparative $C_{\mathrm{T}}$ method [37] was used to analyze the data. The range of expression was calculated using the standard deviation of the $\Delta C_{\mathrm{T}}$ value as described by Applied Biosystems in their "Guide to Performing Relative Quantitation of Gene expression Using Real-Time Quantitative PCR" [38].

\section{Results}

Analysis of ChIP-Seq peaks and validation with quantitative PCR

ChIP assays were performed using frozen cross-linked Brachypodium leaf tissue. The average fragment size of input and anti-GFP ChIP libraries were 371 and $400 \mathrm{bp}$, respectively. The input library had 14.4 million reads and the GFP Ab ChIP library had 5.6 million reads. Over 93\% of the reads were mapped to the Brachypodium genome. The MACS2 (Model-based Analysis of ChIPSeq) program [39] was used to identify enriched regions using a false discovery cutoff of 0.05 . The location of the enriched peaks in the Brachypodium genome are presented in a supplemental table (Additional file 2). Of the 591 regions enriched, $43 \%$ of the peaks were located in genic regions (from $2 \mathrm{~kb}$ upstream of transcription start to $2 \mathrm{~kb}$ downstream of the stop codon including the coding region) (Fig. 1). Of peaks that were in genic regions, $42 \%$ were located only within promoter regions, $33 \%$ were located in promoter regions and in exons or introns, $22 \%$ were located in exons and introns only, and $2 \%$ were located in both promoter regions and introns and exons (Fig. 1). The genes associated with peaks in genic regions that were enriched greater than 10 -fold with a known putative function are presented in Table 1.

To validate the ChIP-Seq results, ChIP-qPCR was performed on two independent paraquat-stressed leaf samples. Regions were chosen to target peaks with a range of fold-enrichments. The results are summarized in Fig. 2, with the ChIP-Seq fold enrichment of each called peak indicated below the graphs. While fold enrichment varied 


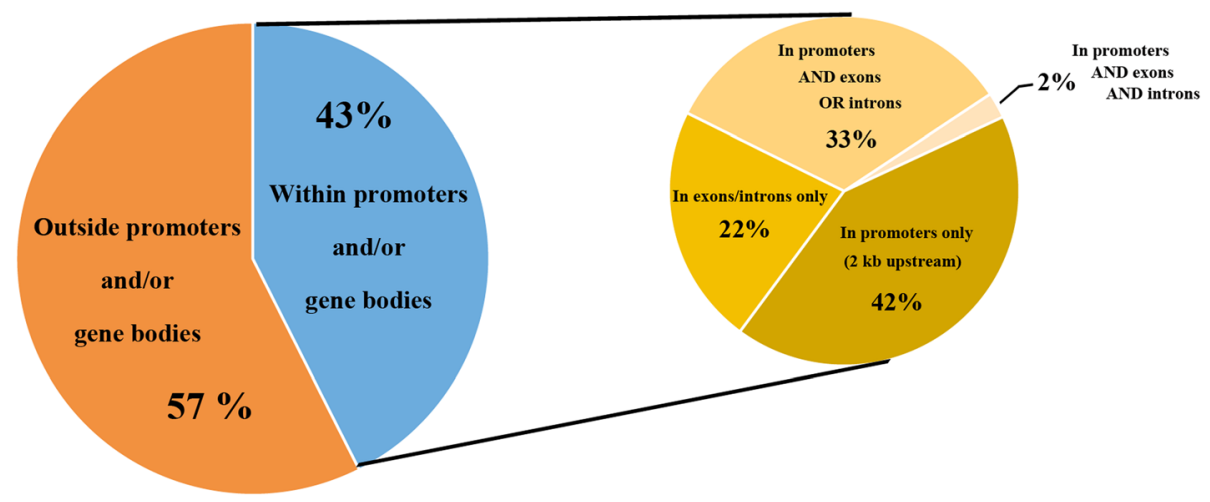

Fig. 1 Distribution of ChIP peaks in the genome. Percent of peaks that reside $2 \mathrm{~kb}$ upstream of the transcriptional start site or $2 \mathrm{~kb}$ downstream of the stop codon (gene body), and location of peaks within the gene bodies

from 1.5-39.3, only peaks that were enriched at least 2.5fold when compared to the input DNA were used for downstream analysis. The ChIP-qPCR values were in general agreement with the ChIP-Seq results.

\section{Motif analysis of bZIP10 target genes}

The most significantly enriched DREME [31] motif was TGDCGACA ( $P$-value of $1.4 \mathrm{e}-33$ and an E-value of 4.6e29) (Fig. 3a), and this motif was distributed evenly among all chromosomes. The next most significant DREME motif (CGGYCGGA) was present mainly in the GC-rich region of chromosome 5 and may be an artifact. Three motifs were identified by MEME [29, 30]. Analysis of the location of the three MEME-identified motifs within the genome revealed that regions associated with MEME Motifs 2 and 3 were located in a $\sim 206 \mathrm{~Kb}$ region on chromosome 5 , between $2.3 \mathrm{~Kb}$ and $209 \mathrm{~Kb}$. While these peaks were all below the 0.05 cutoff $p$-value, this region was notable for being $\mathrm{GC}$-rich, as revealed with sequence motif searches. There is often a GC content bias in ChIP-Seq data [40-42]. At the same time, ChIPSeq peaks have been shown to overlap GC-rich regions of the genome, and there is evidence that transcription factors tend to bind to GC-rich regions, even when they are not near transcription start sites [43]. Interestingly, this region of chromosome 5 contains small regions of homology to heat shock proteins as depicted in the PASA assembled ESTs in the genome browser [44], but there are very few genes that have been annotated in this region of chromosome 5 . However, due to the high GC content in this region (bolded peaks in Additional file 2), these two MEME identified motifs are presumed to be false positives. MEME identified Motif 1 (Fig. 3b) was distributed evenly among all the chromosomes. The peaks identified by MEME and DREME are very similar, but with less discrimination in the central nucleotides in the binding site identified by MEME. Interestingly, none of the motifs found in this study contained the most reported bZIP binding ACGT core sequence, which is present in different bZIP binding sites, including the G-box (CACGTG); C-box (GACGTC); and A-box (TACGTA) motifs reported for bZIP proteins [8, 45]. However, the DREME motif, TGDCGACA, does share homology to the Arabidopsis zinc deficiency response element (RTGTCGACAY) previously reported [13]. The ChIP enriched sequences were examined for the presence of the identified MEME motif or for motifs related to binding sites for other bZIP transcription factors, such as the C, A or G boxes [8, 45]. Several of the peaks enriched greater than 10 -fold did not contain the identified motif, but some did contain ACGT core motifs, suggesting the possibility of heterodimerization between the bZIP10 TF and other bZIP TFs [46].

\section{Gene ontology analysis to identify biological and functional enriched categories}

Gene Ontology (GO) studies using the AgriGO online Gene Set Enrichment Analysis (GSEA) tool (http:// bioinfo.cau.edu.cn/agriGO/analysis.php) [32, 33] revealed $19 \mathrm{GO}$ categories belonging to the Biological Process (BP) ontology that were determined to be significantly over-represented in the ChIP-Seq sample relative to the Brachypodium genome (Additional file 3, Fig. 4). Gene ontology categories significantly enriched ( $p$-value $<0.01$; orange colored boxes in Fig. 4 ) for biological processes included metabolic processes (cellular, primary and macromolecular) and metal ion transport. Additional categories were identified when using a $P$ value greater than 0.01 and less than 0.05 (Fig. 4; yellow boxes). Four biological function categories were significantly enriched with (False Discovery Rates) FDRs and $p$ values < 0.01: metal ion transmembrane transporter, transferase, catalytic, and binding activities (Additional file 3). Several other functional categories including kinases and 
Table 1 List of genes and their putative function

\begin{tabular}{|c|c|c|}
\hline Gene Name & Putative Function & \\
\hline Bradi1g06030 & arachidonic acid binding (Blast2GO) & 18.0 \\
\hline Bradi1g06882 & PTHR31286:SF1 - NUCLEIC ACID BINDING / ZINC ION BINDING PROTEIN-RELATED & 18.2 \\
\hline Bradi1g22650 & leucine-rich repeat protein kinase & 12.4 \\
\hline Bradi1g33347 & PTHR24093:SF272 - CADMIUM/ZINC-TRANSPORTING ATPASE HMA1 & 11.4 \\
\hline Bradi1g35050 & Glycosyl hydrolase (GH) & 10.0 \\
\hline Bradi1g37100 & PF01152 - Bacterial-like globin (Bac_globin) & 25.4 \\
\hline Bradi1g37667 & PTHR1 1040//PTHR1 1040:SF52 - ZINC/IRON TRANSPORTER // SUBFAMILY NOT NAMED & 31.2 \\
\hline Bradi1g48360 & F-Box & 19.7 \\
\hline Bradi1g49020 & PTHR14209//PTHR14209:SF7 - ISOAMYL ACETATE-HYDROLYZING ESTERASE 1 & 12.2 \\
\hline Bradi1g53680 & high affinity zinc uptake transmembrane transporter activity (Blast2GO) & 36.1 \\
\hline Bradi1g61170 & glucosaminyl-phosphotidylinositol O-acyltransferase activity (Blast2GO) & 12.5 \\
\hline Bradi1g68300 & PTHR23310:SF14 - PROTEIN ACBP-7 & 13.0 \\
\hline Bradi1g74270 & PF04720 - PDDEXK-like family of unknown function (PDDEXK_6) & 15.7 \\
\hline Bradi2g07940 & PF05558 - DREPP plasma membrane polypeptide (DREPP) & 15.4 \\
\hline Bradi2g13590 & endopeptidase activity (Blast2GO) & 11.1 \\
\hline Bradi2g19620 & PF04520 - Senescence regulator (Senescence_reg) & 10.9 \\
\hline Bradi2g22520 & zinc ion transmembrane transporter activity (Blast2GO) & 28.6 \\
\hline Bradi2g22530 & high affinity zinc uptake transmembrane transporter activity (Blast2GO) & 31.4 \\
\hline Bradi2g23520 & bHLH transcription factor & 18.0 \\
\hline Bradi2g23560 & PF00582 - Universal stress protein family (Usp) & 11.3 \\
\hline Bradi2g25580 & PTHR31251:SF2 - SQUAMOSA PROMOTER-BINDING-LIKE PROTEIN 7 & 24.1 \\
\hline Bradi2g25677 & PTHR13268:SFO - BREAST CARCINOMA-AMPLIFIED SEQUENCE 3 & 18.7 \\
\hline Bradi2g33110 & zinc ion transmembrane transporter activity (Blast2GO) & 32.3 \\
\hline Bradi2g44850 & 1.14.13.138 - Indolin-2-one monooxygenase / CYP71C2 & 19.1 \\
\hline Bradi2g50840 & K14487 - auxin responsive GH3 gene family (GH3) & 10.2 \\
\hline Bradi2g54670 & GRAS transcription factor & 13.7 \\
\hline Bradi2g59490 & aspartate kinase activity (Blast2GO) & 22.6 \\
\hline Bradi3g06780 & KOG0156 - Cytochrome P450 CYP2 subfamily & 10.6 \\
\hline Bradi3g11300 & Putative glycosyltransferase belonging to CAZy family GT61 & 18.0 \\
\hline Bradi3g17900 & zinc ion transmembrane transporter activity (Blast2GO) & 39.2 \\
\hline Bradi3g28990 & PTHR33083:SF9 - EMB & 13.2 \\
\hline Bradi3g41600 & PF07712 - Stress up-regulated Nod 19 (SURNod19) & 10.6 \\
\hline Bradi3g47530 & PF04570 - zinc-finger of the FCS-type & 17.6 \\
\hline Bradi4g03530 & RING, subfamily zinc finger (C3HC4-type RING finger) family protein & 11.5 \\
\hline Bradi4g07210 & 1.1.99.35 - Soluble quinoprotein glucose dehydrogenase / Soluble glucose dehydrogenase & 10.8 \\
\hline Bradi4g12190 & phospholipid:diacylglycerol acyltransferase activity & 11.1 \\
\hline Bradi4g25880 & CAMK_KIN1/SNF1/Nim1_like.31 - CAMK includes calcium/calmodulin dependent protein kinases & 12.0 \\
\hline Bradi4g42530 & cation transmembrane transporter activity & 14.1 \\
\hline Bradi4g44460 & CAMK_KIN1/SNF1/Nim1_like.35 - CAMK includes calcium/calmodulin dependent protein kinases & 12.3 \\
\hline Bradi5g18277 & Prolyl oligopeptidase / Prolyl endopeptidase & 30.3 \\
\hline
\end{tabular}

The genes listed in this table are limited to those associated with peaks that were enriched greater than 10-fold and have been classified with a known function

phosphotransferases, cation/ion transmembrane transporters, transferases (phosphorus-containing and glycosyl groups), and some nucleoside/nucleotide binding activities were also enriched with $P$ values between 0.01 and 0.05 . Members of each enriched GO category are listed in Additional file 3. 

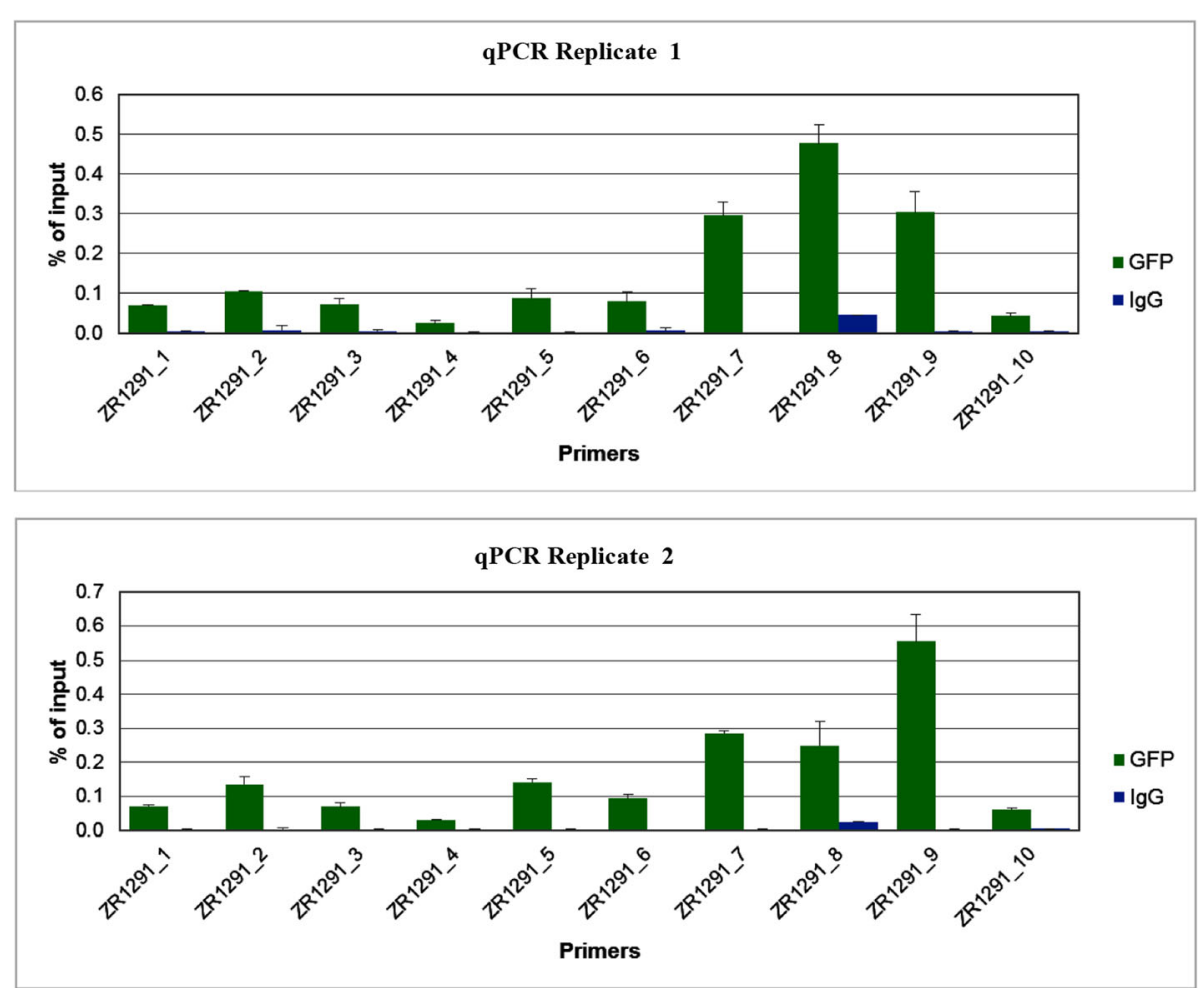

$$
\begin{array}{ccccccccccc} 
& \multicolumn{1}{c}{\text { ChIP Seq Fold Enrichment }} \\
\text { Primer } & \mathbf{1} & \mathbf{2} & \mathbf{3} & \mathbf{4} & \mathbf{5} & \mathbf{6} & \mathbf{7} & \mathbf{8} & \mathbf{9} & \mathbf{1 0} \\
\hline \mathbf{6} & \mathbf{8} & \mathbf{8 . 6} & \mathbf{9 . 1} & \mathbf{9 . 2} & \mathbf{1 1 . 6} & \mathbf{1 4 . 4} & \mathbf{2 7 . 3} & 39.4 & 4.9
\end{array}
$$

Fig. 2 Verification of ChIP-Seq data with ChIP-qPCR. Two independent immunoprecipitates were analyzed with qPCR and compared to the ChIP-Seq data. The fold enrichment from the ChIP-Seq data is provided in red at the bottom of the image

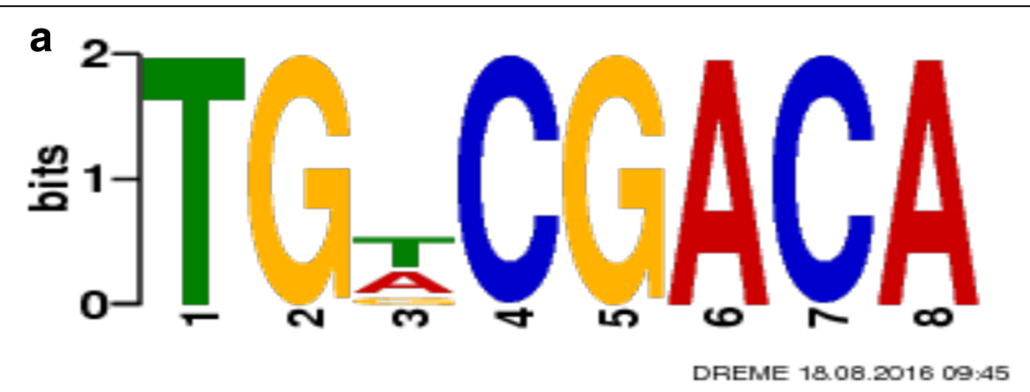

b

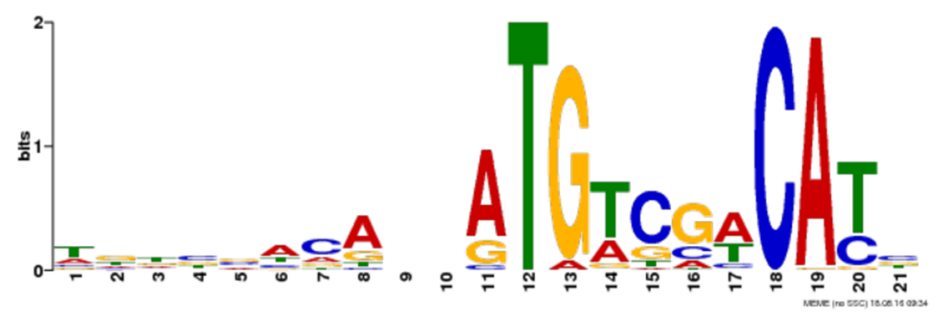

Fig. 3 Consensus BdbZIP10-binding motif. The consensus sequences were determined by DREME (Discriminative Regular Expression Motif Elicitation; [2]) and MEME (Multiple Em for Motif Elicitation; [29]). Motifs identified with DREME (a) and MEME (b). The size of the letters in the figures is proportional to the frequency of the nucleotide in the motif 


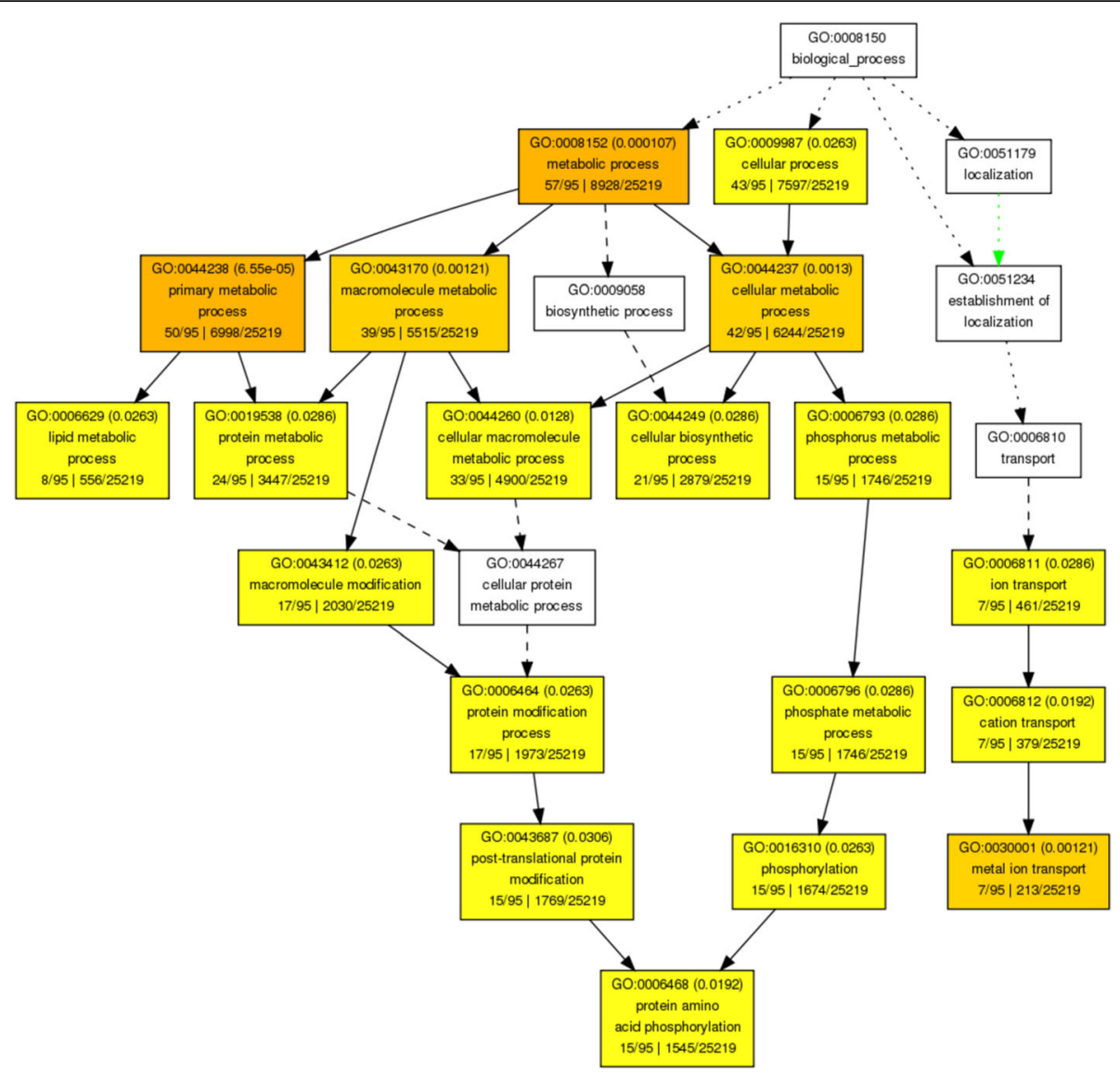

Fig. 4 Functional categories of BdbZIP10-bound genes. Each square represent a single gene ontology class. White boxes were not significantly enriched. Yellow boxes represent groups of genes that are significantly enriched at $0.01<P<0.05$. Orange boxes are significant at $P<0.01$

\section{Expression analysis of genes from enriched Gene Ontology (GO) categories}

Expression levels of several genes from the enriched GO categories with ChIP-fold enrichment greater than ten were analyzed in the presence (paraquat treated) or absence (water treated) of oxidative stress in BdbZIP10GFP OE and WT plants (Table 2). Since a value of one represents equivalent expression, ranges that surrounded, but included one, were not considered to have significantly different expression. Five genes related to zinc transport were analyzed and four of these genes were found to be up-regulated in nonstressed BdZIP10 OE compared to nonstressed WT plants (Bradi2g33110. 1, Bradi2g22520.1, Bradilg53680.1, Bradi3g17900.1), while expression of Bradi5g21580.1 was similar in nonstressed WT and BdbZIP10 OE plants (Table 2). These zinc transporter genes were down regulated in BdbZIP10-GFP OE plants treated with paraquat compared to the transgenic control treatment, but were variable in their response in WT plants treated with paraquat compared to control WT plants; Bradi2g33110. 1 was down regulated, Bradilg53680.1 tended towards down regulation, and Bradi2g22520.1 was upregulated, while the other genes had equivalent expression. All of these genes, except Bradi5g21580.1, were increased in BdbZIP10-GFP OE plants when compared to WT under both control and oxidative stress conditions. Expression of Bradi2g22520.1 and Bradilg53680.1, both having zinc ion transmembrane transporter activity, were increased over 30 fold in BdbZIP10-GFP OE plants compared to WT under control conditions. Some genes, such as Bradilg68300 (Acyl-CoA-Binding protein) were similarly induced in both the BdbZIP10-GFP OE and WT under oxidative stress (paraquat treatment), but showed no expression differences between transgenic and WT plants under stressed and control conditions (Table 2). Similarly, a stress up-regulated Nod19 gene was increased in both BdbZIP10-GFP OE and WT plants when exposed to paraquat, with slightly higher induction in the WT plants; and slightly increased expression in BdbZIP10-GFP OE plants compared to WT plants under control conditions (Table 2). A bacterial-like globin gene (Bradilg37100) showed slightly reduced expression in the transgenic plants when treated with paraquat compared to transgenics treated with water, and was expressed at higher levels in the BdbZIP10-GFP OE 
Table 2 Expression analysis of select genes

\begin{tabular}{|c|c|c|c|c|c|}
\hline \multirow[b]{2}{*}{ Gene Name } & \multicolumn{2}{|c|}{$\begin{array}{l}\text { Paraquat treatment } \\
\text { compared to water }\end{array}$} & \multicolumn{2}{|c|}{$\begin{array}{l}\text { BdbZIP10-GFP OE } \\
\text { Compared to WT }\end{array}$} & \multirow[t]{2}{*}{$\begin{array}{l}\text { Putative function } \\
\text { Phytozome/Panther }\end{array}$} \\
\hline & BdbZIP10-GFP OE & WT & Water & Paraquat & \\
\hline Bradi2g33110.1 & $0.2-0.48$ & $0.24-0.33$ & $2.0-4.1$ & $2.1-4.9$ & Bradi2g33110.1.p - zinc ion transmembrane transporter activity \\
\hline Bradi2g22520.1 & $0.28-0.62$ & $2.1-2.8$ & $52.8-93.1$ & $8.1-18.2$ & Zinc ion transmembrane transporter activity \\
\hline Bradi1g53680.1 & $0.05-0.14$ & $0.24-1.17$ & $30-123$ & $5.4-18.1$ & $\begin{array}{l}\text { High affinity zinc uptake } \\
\text { transmembrane transporter activity }\end{array}$ \\
\hline Bradi3g17900.1 & $0.27-0.68$ & $0.6-1.2$ & $6.4-14.8$ & $3.1-7.8$ & $\begin{array}{l}\text { Zinc ion transmembrane } \\
\text { transporter activity }\end{array}$ \\
\hline Bradi5g21580.1 & $0.16-0.25$ & $0.8-1.2$ & $1.1-2.4$ & $1.0-1.4$ & $\begin{array}{l}\text { High affinity zinc uptake } \\
\text { transmembrane transporter activity }\end{array}$ \\
\hline Bradi1g68300 & $3.9-5.2$ & $3.9-5.1$ & $0.8-1.2$ & $0.9-1.3$ & PTHR23310:SF14-Protein ACBP-7 \\
\hline Bradi1g37100 & $0.59-0.81$ & $0.72-1.63$ & $2.7-5.9$ & $2.1-3.0$ & PF01152 - Bacterial-like globin; Thoredoxin peroxidase \\
\hline Bradi1g49020 & $0.4-0.6$ & $3.3-7.4$ & $3.4-8.0$ & $0.4-0.6$ & PTHR14209//PTHR14209:SF7- Isoamyl acetate-hydrolyzing esterase 1 \\
\hline Bradi3g41600 & $3.9-5.2$ & $7.8-13.1$ & $1.2-1.7$ & $0.5-0.7$ & $\begin{array}{l}\text { Stress up-regulated Nod19 } \\
\text { (SURNod19) }\end{array}$ \\
\hline
\end{tabular}

Comparison of gene expression levels between paraquat and water treated BdbZIP10 OE and wildtype plants. Gene expression level of BdbZIP10-GFP OE compared to wildtype plants. Numbers indicate the range of three independently BdbZIP10-GFP OE transformed lines, calculated using the standard deviation of the $C_{t}$ values. Bold text indicates the most significant changes between BdbZIP10-GFP OE and wildtype plants

plants compared to the WT under both control and paraquat conditions (Table 2). Expression of an isoamyl acetate-hydrolyzing esterase (Bradi1g49020) was increased in WT plants and slightly decreased in BdbZIP10-GFP OE plants treated with paraquat compared to untreated plants. Transgenic BdbZIP10-GFP OE plants had increased isoamyl acetate-hydrolyzing esterase expression levels compared to WT plants in the control treatment and slightly decreased levels with paraquat treatment (Table 2).

\section{Discussion}

Zinc is an essential micronutrient in many cellular processes, as a cofactor for many enzymes and transcription factors, as a structural component of proteins, and as a signaling molecule. It is also thought to be involved in protecting the cell from oxidative stress [47, 48]. It is therefore essential that organisms have the capacity to maintain zinc homeostasis within the cell and within the organism through zinc uptake, transport, storage, exclusion and/or compartmentalization [49].

Paraquat enters plant cells, then chloroplasts, via plasma membrane-bound homologs of polyamine transporters, where it strongly induces oxidative stress by production of reactive oxygen species (Fig. 5) [50]. Accumulation of ROS initiates an abiotic stress response, including such phosphorylation-based signal transduction pathways as mitogen-acitvated protein kinases (MAPKs) and cyclindependent protein kinases (CDPKs) [51]. Signal transduction leads to the activation of transcription factors that upregulate expression of key stress response genes.

Superoxide dismutases (SODs) constitute an important part of the plant cell's defense against ROS. SODs are categorized into three groups according to their metal cofactors (iron, manganese, copper-zinc), and those in the copper-zinc category are found throughout the plant cell [52]. Therefore, the abiotic stress response and zinc homeostasis are closely connected.

In Arabidopsis, two transcription factors, AtbZIP19 and AtbZIP23, have been reported to regulate the zinc deficiency response [13], but a later report indicated that the single Atbzip19 mutant was hypersensitive to zinc deficiency, and they concluded that AtbZIP19 is involved in response to $\mathrm{Zn}$ deficiency, while AtbZIP23 is important for maintaining $\mathrm{Zn}$ homeostasis [14]. They found two $\mathrm{Zn}$ transporters and three defensin-like family proteins that were up-regulated in WT compared to Atbzip19 mutants on zinc deficient media, and they suggested that these proteins are responsible for the hypersensitivity of the Atbzip19 mutant to zinc deficiency [14].

An excess of zinc is also detrimental to plants, but there are species such as Arabidopsis halleri (L.) O'Kane and Al-Shehbaz ssp. halleri that are Zn hyperaccumulators and are able to grow in metal-contaminated soils $[53,54]$. Studies comparing $A$. halleri to $A$. thaliana revealed high constitutive expression of genes involved in metal homeostasis. These included genes coding for ZIP transporter proteins and proteins that may function in cellular $\mathrm{Zn}$ sequestration and detoxification such as putative P-type metal ATPase, cation diffusion facilitator, and nicotianamine synthase genes $[55,56]$. Plant defensins were also reported to play a role in tolerance to excess $\mathrm{Zn}$ in the $\mathrm{Zn}$ hyper-accumulator Arabidopsis halleri and it was suggested they may play a role in metal physiology [57]. These genes are likely essential for maintaining zinc homeostasis in plants. 


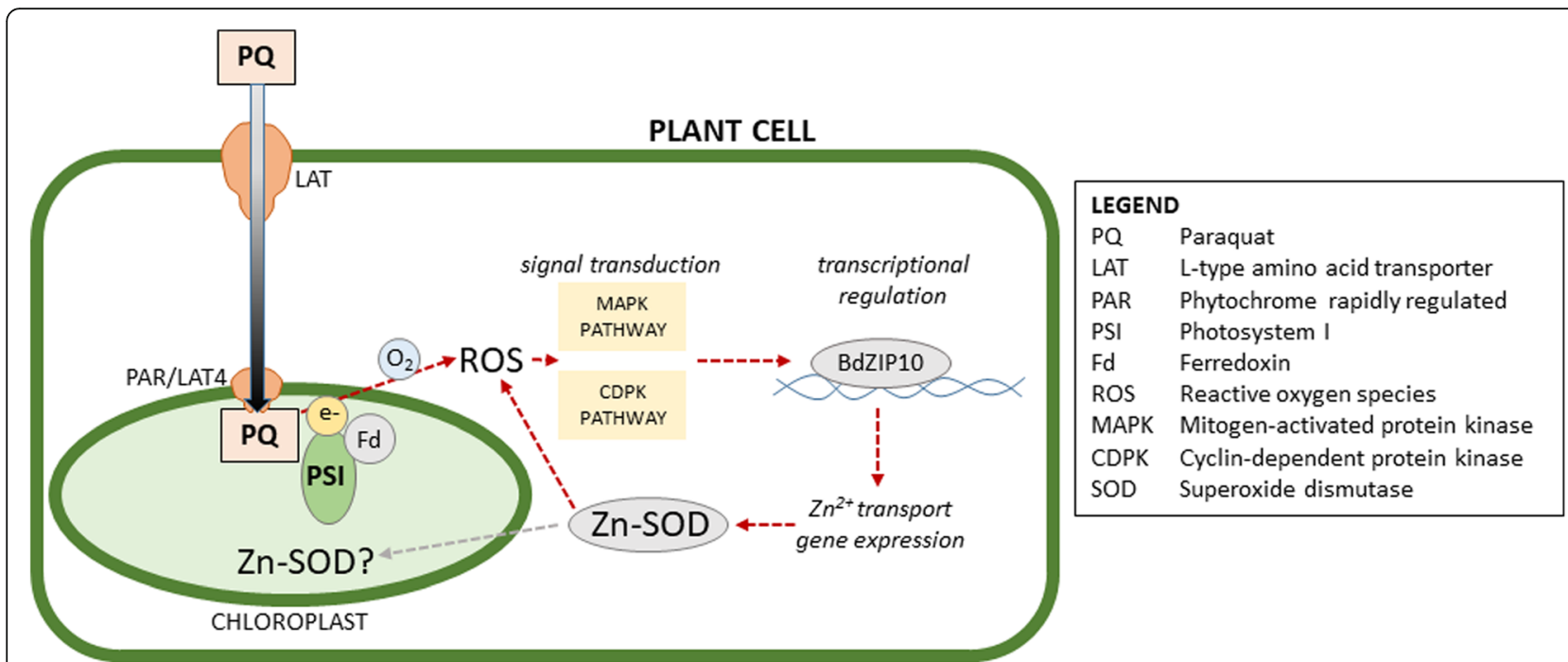

Fig. 5 Proposed role of BdbZIP10 in response to paraquat-induced oxidative stress. Paraquat entry into plant chloroplasts results in production of reactive oxygen species, which in turn initiate protein kinase signaling pathways. Phosphorylation cascades activate transcription factors such as BdbZIP10. Overexpression of BdbZIP10 further upregulates genes controlling intracellular zinc transport, increasing activity of cytosolic and/or chloroplastic superoxide dismutases that have $\mathrm{Zn}^{2+}$ as a cofactor

Overexpression of a Brachypodium homolog of AtbZIP19/23, BdbZIP10, enhanced oxidative stress resistance in Brachypodium, likely through the activation of detoxification and protective oxidative stress genes [17]. We performed ChIP sequence analysis to identify binding motifs and potential target genes of BdbZIP10 when plants were exposed to paraquat-induced oxidative stress. A binding motif similar to the zinc deficiency response element (ZDRE) was identified (Fig. 3), which is not unexpected, as Brachypodium BdbZIP10 is most homologous to AtbZIP 19 and 23, which have been shown to bind to the ZDRE. Many of the target genes identified are involved in zinc homeostasis. Five genes with zinc transporter activity were shown to be highly enriched in the ChIP sequences, but not all of these showed differential expression between WT and BdbZIP10-GFP OE plants (Tables 1 and 2). Data available for these zinc transporter genes in Phytozome [44] revealed that most were also found to be differentially expressed during some other type of stress - cold, salt, drought, or heat stress; except one of the zinc ion transporters (Bradi2g33110.1), which was differentially expressed in root tissues exposed to different nitrogen compounds [44]. It is possible that the zinc transporter genes that were not upregulated in response to paraquat-induced oxidative stress could require additional TFs for induction, may be localized in the roots, or could be induced in response to zinc deficiency, but not oxidative stress. It will be interesting to look at the effect of high and low levels of zinc in BdbZIP10 OE plants to determine if a subset of zinc transporter genes might be differentially regulated during zinc stress, as compared those identified during oxidative stress. A Cd/ Zn-transporting ATPase HMA1 was also present in the ChIP-Seq enriched peaks (Table 1). This protein is localized in the chloroplast and is thought to control $\mathrm{Zn}$ content in plastids, is involved in $\mathrm{Cd}$ detoxification, and functions as a Ca/heavy metal pump [58]. Furthermore, Arabidopsis HMA1 knockout plants were more sensitive to Zn than WT plants [59], suggesting the importance of this enzyme in $\mathrm{Zn}$ homeostasis. An Acyl CoA binding protein was also among the ChIP-enriched sequences. Besides their role in phospholipid metabolism and signaling, it has been suggested that members of this class of proteins may also play a role in heavy metal binding [60]. The fact that this protein was induced in both WT and BdbZIP10-GFP OE plants when exposed to paraquat may indicate that it plays a role in signaling during oxidative stress.

There were several different classes of compounds involved in oxido-reductase reactions that were enriched in the ChIP-Seq genes. A bacterial-like globin gene/ thioredoxin peroxidase, which interacts with oxygen was also identified (Table 1) and found to be upregulated in the BdbZIP10-GFP OE compared to WT plants under both stressed and control conditions (Table 2). A stress up-regulated Nod 19 gene that was previously shown to be important in beans to cope with abiotic and biotic generated oxidative stress [61] was enriched in the ChIP-Seq peaks. This gene was found to be upregulated in both the WT and BdbdbZIP10-GFP OE plants when exposed to paraquat treatment, but there was little difference between the transgenic lines and the WT plants under both control and paraquat conditions. 
Cytochrome P450s, which are involved in assorted biosynthetic pathways and in detoxification of multiple classes of compounds including heavy metals and herbicides [62], were also identified in our ChIP-Seq library, however, the identified MEME motif was not present in the promoter of this gene. Several other genes also lacked the ZDRE-like motif and did not contain any of the other bZIP binding motifs. It is possible that BdbZIP10 interacts with other TFs/signals present during oxidative stress not related to this motif, or alternatively this could be an artifact.

There were also some genes that were ChIP-Seq enriched that are not directly involved in zinc transport, but are related to lipid metabolism and gene regulation. A putative SF7-Isoamyl acetate-hydrolyzing esterase (Pfam: GDSL-like Lipase/Acylhydrolase) was upregulated in the absence of paraquat in the BdbZIP10-GFP OE plants compared to WT plants, and was also induced in WT when exposed to paraquat (Table 2). Many GDSL esterase/lipases are involved in biotic and abiotic stress responses, and members of this family are also involved in plant growth and development and defense responses [63]. This gene did not contain the identified MEME binding site, but it did contain an ACGT motif binding site. Another gene coding for an enzyme involved in triacylglycerol (TAG) synthesis was present in the ChIP-Seq enriched genes (Bradi4g12190, phospholipid:diacylglycerol acyltransferase), but there were no bZIP binding sites in the ChIPed peak sequence, suggesting this may be an artifact, or that there are alternative binding sites/interactions at play. Triacylglycerol synthesis is involved in maintaining membrane lipid homeostasis [64] and protecting cells from oxidative damage during lipid peroxidation $[65,66]$. It would be an interesting feedback mechanism if a zinc deficiency TF helps to regulate genes that are involved in membrane stability, as zinc deficiency leads to oxidative stress and eventually membrane destabilization.

\section{Conclusion}

The BdbZIP10 (Bradi1g30140) protein from Brachypodium is most closely related to the Group 1 members of the F family of bZIP transcription factors represented by AtbZIP19 and 23. Members of this group of TFs, the ZIP transporters to which they bind, and the zinc deficiency responsive element (ZDRE) are highly conserved across land plants, suggesting they play a vital role in maintaining zinc homeostasis in plants $[12,13]$. Members of F subgroup 2, which includes AtbZIP24, are more variable across land plants, with monocots generally having more members, but with some plant species lacking members in this subgroup [12]. The conservation across species of bZIP F group 1 members and their relatively higher levels of expression suggest they may serve an essential role in land plants. Members of the bZIP F group 1 are involved in maintaining zinc homeostasis in plants. Zinc deficiency causes decreased crop yields, reduced crop nutritional value, and also contributes to major health problems in humans [47, 48]. Zinc is an important cofactor for many enzymes and transcription factors, including SODs that detoxify ROS during the abiotic stress response (52). Zinc deficiency results in oxidative stress in animal and plant systems $[48,49,67]$. In this study, we identified potential targets of the BdbZIP10 TF in response to paraquat-induced oxidative stress and found a motif that is different from most reported bZIP TF motifs, but with strong homology to the Arabidopsis ZDRE. This, coupled with the fact that our ChIP analysis identified a number of zinc transporter genes suggests that BdbZIP10 is potentially involved in zinc homeostasis, as it relates to oxidative stress. Overexpression of BdbZIP10 may boost cellular $\mathrm{Cu}-\mathrm{Zn}-\mathrm{SOD}$ activity, increasing protection against ROS production. TF motifs play a key role in transcriptional regulation. This study contributes to a better understanding of the role BdbZIP10 plays in oxidative stress in Brachypodium. To truly understand an organism's response to any particular signal or stress, it is crucial to identify the control elements involved in the signaling and response pathways. In addition to the zinc transporter genes, other genes associated with signal transduction pathways, such as phosphatases and kinases were also identified in our ChIP analysis. It will be interesting to investigate the role that these proteins play in the signaling pathways associated with or regulated by BdbZIP10. Our future research will focus on determining the role BdbZIP10 plays in other abiotic stresses.

\section{Additional files}

Additional file 1: Primers used in this study. Primers used for gene expression analysis and their calculated efficiency. Zymo Primers used for ChIP-qPCR analysis. (DOCX $41 \mathrm{~kb}$ )

Additional file 2: List of enriched peaks and their location in the Brachypodium genome. (XLSX $65 \mathrm{~kb}$ )

Additional file 3: Gene ontology categories and their gene members represented in ChIP sequences. Gene ontology (GO) groups, based on biological processes and molecular functions, with False Discovery Rates (FDR) $<0.01$ are bolded, FDR $>0.01$ and $<0.05$ are italicized, and FDR $>$ 0.05 and $<1$ and $P$ values $<0.05$ are in normal font. (DOCX 27 kb)

\section{Abbreviations}

At: Arabidopsis thaliana; Bd: Brachypodium; ChIP: chromatin immunoprecipitation; $C_{T}$ : cycle threshold; DREME: Discriminative Regular Expression Motif Elicitation; GFP: Green fluorescent protein; GO: Gene Ontology; MEME: Multiple EM for Motif Elicitation; RT: reverse transcriptase; Seq: sequencing; TF: transcription factor

\section{Acknowledgements}

Special thanks are extended to Thomas Lockwood (USDA-ARS, Corvallis Oregon) for his assistance in laboratory and greenhouse experiments, and to Dr. Kira Glover-Cutter for her contribution to the original characterization of the Brachypodium bZIP10-GFP OE lines used in this research. 


\section{Funding}

This work was possible through base funding from USDA ARS National Program 215 to the Forage Seed and Cereal Research Unit in Corvallis OR. USDA scientists and collaborators were responsible for designing the study, performing the experiments, analyzing the results, interpreting the data, and writing the manuscripts. A collaboration with Kelly Vining was established by the USDA scientists to supply bioinformatics expertise.

\section{Availability of data and materials}

All data generated are included in this published article and its supplementary files.

\section{Authors' contributions}

RCM and JED were responsible for designing and performing experiments, $\mathrm{KV}$ was responsible for the bioinformatics, and all three authors contributed to writing the manuscript. All authors read and approved the final manuscript.

\section{Ethics approval and consent to participate}

Original seeds of Brachypodium distachyon were obtained from Dr. John Vogel who was working at the USDA-ARS Western Regional Research Center, Genomics and Gene Discovery Research Unit at the time. Experimental methods performed in this research complied with current laws and regulations of the U.S.A. The use of trade, firm, or corporation names in this publication is for the information and convenience of the reader. Such use does not constitute an official endorsement or approval by the United States Department of Agriculture or the Agricultural Research Service of any product or service to the exclusion of others that may be suitable. Relevance to ARS National Programs. NP215: Rangeland, Pasture and Forages: Component "Plant Resources". NP302: Plant Biological and Molecular Processes: Component "Biological Processes that Determine Plant Productivity and Quality."

\section{Consent for publication}

Reviewed and approved for publication by Dr. Ryan Hayes, Research Leader at USDA ARS Forage Seed and Cereal Research Unit.

\section{Competing interests}

The authors declare that they have no competing interests.

\section{Publisher's Note}

Springer Nature remains neutral with regard to jurisdictional claims in published maps and institutional affiliations.

\section{Author details}

${ }^{1}$ USDA ARS National Forage Seed and Cereal Research Unit, 3450 SW Campus Way, Corvallis, OR 97330, USA. ²Department of Horticulture, 4123 Agricultural \& Life Sciences, Oregon State University, Corvallis, OR 97330, USA.

Received: 15 September 2017 Accepted: 29 March 2018 Published online: 10 April 2018

\section{References}

1. Cheatle Jarvela AM, Hinman VF. Evolution of transcription factor function as a mechanism for changing metazoan developmental gene regulatory networks. EvoDevo. 2015;6(1):3. https://doi.org/10.1186/2041-9139-6-3.

2. Moreno-Risueno MA, Martinez M, Vicente-Carbajosa J, Carbonero P. The family of DOF transcription factors: from green unicellular algae to vascular plants. Mol Gen Genomics. 2007;277:379-90. https://doi.org/10.1007/s00438-006-0186-9.

3. Martinez-Castilla LP, Alvarez-Buylla ER. Adaptive evolution in the Arabidopsis MADS-box gene family inferred from its complete resolved phylogeny. Proc Natl Acad Sci U S A. 2003;100:13407-12. https://doi.org/10.1073/pnas. 1835864100.

4. Deppmann CD, Alvania RS, Taparowsky EJ. Cross-species annotation of basic leucine zipper factor interactions: insight into the evolution of closed interaction networks. Mol Biol Evol. 2006;23:1480-92.

5. Corrêa LGG, Riaño-Pachón DM, Schrago CG, dos Santos RV, Mueller-Roeber $B$, Vincentz $M$. The role of bZIP transcription factors in green plant evolution: adaptive features emerging from four founder genes. PLoS One. 2008;3(8):e2944.

6. Nijhawan A, Jain M, Tyagi AK, Khurana JP. Genomic survey and gene expression analysis of the basic leucine zipper transcription factor family in rice. Plant Physiol. 2008;146:333-50.
7. Lindemose S, O'Shea C, Jensen MK, Skriver K. Structure, function and networks of transcription factors involved in abiotic stress responses. Int J Mol Sci. 2013;14(3):5842-78.

8. Jakoby M, Weisshaar B, Dröge-Laser W, Vicente-Carbajosa J, Tiedemann J, Kroj T, Parcy F. bZIP transcription factors in Arabidopsis. Trends Plant Sci. 2002;7(3):106-11.

9. Liu J, Chen N, Chen F, Cai B, Dal Santo S, Tornielli GB, Pezzotti M, Cheng ZM. Genome wide analysis and expression profile of the bZIP transcription factor gene family in grapevine (Vitis vinifera). BMC Genomics. 2014;15:281. https://doi.org/10.1186/1471-2164-15-281.

10. Liao Y, Zou HF, Wei W, Hao YJ, Tian AG, Huang J, Liu YF, Zhang JS, Chen SY. Soybean GmbZIP44 GmbZIP62 and GmbZIP78 genes function as negative regulator of ABA signaling and confer salt and freezing tolerance in transgenic Arabidopsis. Planta. 2008;228(2):225-40. https://doi.org/10.1007/ s00425-008-0731-3.

11. Liu X, Chu Z. Genome-wide evolutionary characterization and analysis of bZIP transcription factors and their expression profiles in response to multiple abiotic stresses in Brachypodium distachyon. BMC Genomics. 2015; 16(1):227.

12. Castro PH, Lilay GH, Muñoz-Mérida A, Schjoerring JK, Azevedo H, Assunção AGL. Phylogenetic analysis of F-bZIP transcription factors indicates conservation of the zinc deficiency response across land plants. Sci Rep. 2017;7:3806. https://doi.org/10.1038/s41598-017-03903-6.

13. Assunção AGL, Herrero E, Lin YF, Huettel B, Talukdar S, Smaczniak C, Immink RGH, van Eldik M, Fiers M, Schat H, Aarts MGM. Arabidopsis thaliana transcription factors bZIP19 and bZIP23 regulate the adaptation to zinc deficiency. Proc Natl Acad Sci U S A. 2010;107:10296-301.

14. Inaba S, Kurata R, Kobayashi M, Yamagishi Y, Mori I, Ogata Y, Fukao Y. Identification of putative target genes of bZIP19, a transcription factor essential for Arabidopsis adaptation to Zn deficiency in roots. Plant J. 2015; 84(2):323-34. https://doi.org/10.1111/tpj.12996.

15. Yang O, Popova OV, Süthoff U, Lüking I, Dietz KJ, Golldack D. The Arabidopsis basic leucine zipper transcription factor AtbZIP24 regulates complex transcriptional networks involved in abiotic stress resistance. Gene. 2009;436:45-55

16. Sharma PN, Kumar P, Tewari RK. Early signs of oxidative stress in wheat plants subjected to zinc deficiency. J Plant Nutr. 2004;27(3):451-63. https:// doi.org/10.1081/PLN-120028873.

17. Glover-Cutter KM, Alderman S, Dombrowski JE, Martin RC. Enhanced oxidative stress resistance through activation of a zinc deficiency transcription factor in Brachypodium distachyon. Plant Physiol. 2014;166(3):1492-505.

18. Dodge AD. The mode of action of the bipyridylium herbicides, paraquat and diquat. Endeavour. 1971:30:130-5.

19. Mittler R. Oxidative stress, antioxidants and stress tolerance. Trends Plant Sci. 2002;7(9):405-10.

20. Johnson DS, Mortazavi A, Myers RM, Wold B. Genome-wide mapping of in vivo protein-DNA interactions. Science. 2007:316:1497-502.

21. Robertson G, Hirst M, Bainbridge M, Bilenky M, Zhao Y, Zeng T, Euskirchen G, Bernier B, Varhol R, Delaney A, Thiessen N, Griffith OL, He A, Marra M, Snyder M, Jones S. Genome-wide profiles of STAT1 DNA association using chromatin immunoprecipitation and massively parallel sequencing. Nat Methods. 2007:4:651-7.

22. Gleave AP. A versatile binary vector system with a T-DNA organisational structure conducive to efficient integration of cloned DNA into the plant genome. Plant Mol Biol. 1992;20:1203-7.

23. Lazo GR, Stein PA, Ludwig RA. A DNA transformation-competent Arabidopsis genomic library in agrobacterium. Biotechnology. 1991;9:963-7.

24. Zhang A, Jiang M, Zhang J, Tan M, Hu X. Mitogen-activated protein kinase is involved in abscisic acid-induced antioxidant defense and acts downstream of reactive oxygen species production in leaves of maize plants. Plant Physiol. 2006;141:475-87.

25. Wan X, Tan J, Lu S, Lin C, Hu Y, Guo Z. Increased tolerance to oxidative stress in transgenic tobacco expressing a wheat oxalate oxidase gene via induction of antioxidant enzymes is mediated by $\mathrm{H}_{2} \mathrm{O}_{2}$. Physiol Plant. 2009;136:30-44.

26. Langmead B, Trapnell C, Pop M, Salzberg SL. Ultrafast and memory-efficient alignment of short DNA sequences to the human genome. Genome Biol. 2009;10(3):R25.

27. Kent WJ, Zweig AS, Barber G, Hinrichs AS, Karolchik D. BigWig and BigBed: enabling browsing of large distributed data sets. Bioinformatics. 2010;26(17):2204-7.

28. Feng J, Liu T, Zhang Y. Using MACS to identify peaks from ChIP-Seq data. Current protocols in bioinformatics, chapter 2; 2011. p. 2-14. 
29. Machanick P, Bailey TL. MEME-ChIP: motif analysis of large DNA datasets. Bioinformatics. 2011;27(12):1696-7.

30. Bailey $\mathrm{TL}$, Elkan C. Fitting a mixture model by expectation maximization to discover motifs in biopolymers, Proceedings of the second international conference on intelligent Systems for Molecular Biology. Menlo Park: AAAI Press; 1994. p. 28-36.

31. Bailey TL. DREME: motif discovery in transcription factor ChIP-seq data. Bioinformatics. 2011;27(12):1653-9. https:/doi.org/10.1093/bioinformatics/btr261.

32. Du Z, Zhou X, Ling Y, Zhang Z, Su Z. AgriGO: a GO analysis toolkit for the agricultural community. Nucl Acids Res. 2010;38:W64-70.

33. Tian T, Liu Y, Yan H, You Q, Yi X, Du Z, Xu W, Su Z. AgriGO v2.0: a GO analysis toolkit for the agricultural community, 2017 update. Nucleic Acids Res. 2017;45:W122-9. https://doi.org/10.1093/nar/gkx382.

34. Benjamini $Y$, Yekutieli D. The control of the false discovery rate in multiple testing under dependency. Ann Statist. 2001:29(4):1165-88.

35. Arvidsson S, Kwasniewski M, Riano-Pachon DM, Mueller-Roeber B. QuantPrime - a flexible tool for reliable high-throughput primer design for quantitative PCR. BMC Bioinformatics. 2008;9(1):465

36. Hong SY, Seo PJ, Yang MS, Xiang F, Park CM. Exploring valid reference genes for gene expression studies in Brachypodium distachyon by real-time PCR. BMC Plant Biol. 2008:8(1):112.

37. Livak KJ, Schmittgen TD. Analysis of relative gene expression data using real-time quantitative PCR and the $2^{-\Delta \Delta C t}$ method. Methods. 2001;25(4):4028. https://doi.org/10.1006/meth.2001.1262.

38. Applied Biosystems. Guide to performing relative quantification of gene expression using real-time quantitative PCR. p. 56-9. www3. appliedbiosystems.com/cms/groups/mcb_support/documents/ generaldocuments/cms_042380.pdf

39. Zhang Y, Liu T, Meyer CA, Eeckhoute J, Johnson DS, Bernstein BE, Nusbaum C, Myers RM, Brown M, Li W, Liu XS. Model-based analysis of ChIP-Seq (MACS). Genome Biol. 2008;9(9):R137. https://doi.org/10.1186/gb-2008-9-9-r137.

40. Benjamini Y, Speed TP. Summarizing and correcting the GC content bias in high-throughput sequencing. Nucleic Acids Res. 2012;4(10):e72.

41. Dohm JC, Lottaz C, Borodina T, Himmelbauer H. Substantial biases in ultrashort read data sets from high-throughput DNA sequencing. Nucleic Acids Res. 2008;36(16):e105.

42. Ramachandran P, Palidwor GA, Perkins TJ. BIDCHIPS: bias decomposition and removal from ChIP-seg data clarifies true binding signal and its functional correlates. Epigenetics Chromatin. 2015;8(1):33.

43. Wang J, Zhuang J, lyer S, Lin X, Whitfield TW, Greven MC, Pierce BG, Dong $X$, Kundaje A, Cheng $Y$, Rando OJ. Sequence features and chromatin structure around the genomic regions bound by 119 human transcription factors. Genome Res. 2012;22(9):1798-812.

44. Goodstein DM, Shu S, Howson R, Neupane R, Hayes RD, Fazo J, Mitros T, Dirks W, Hellsten U, Putnam N, Rokhsar DS. Phytozome: a comparative platform for green plant genomics. Nucleic Acids Res. 2011;40:D1178-86. https://doi.org/10.1093/nar/gkr944.

45. Foster R, Izawa T, Chua N. Plant bZIP proteins gather at ACGT elements. FASEB J. 1994;8(2):192-200.

46. Ellenberger TE, Brandl CJ, Struhl K, Harrison SC. The GCN4 basic region leucine zipper binds DNA as a dimer of uninterrupted alpha helices: crystal structure of the protein-DNA complex. Cell. 1992;71(7):1223-37.

47. Cakmak I. Tansley review no. 111 possible roles of zinc in protecting plant cells from damage by reactive oxygen species. New Phytol. 2000;146(2):185-205.

48. Marreiro DD, Cruz KJ, Morais JB, Beserra JB, Severo JS, de Oliveira AR. Zinc and oxidative stress: current mechanisms. Antioxidants. 2017;6(2):24.

49. Broadley MR, White PJ, Hammond JP, Zelko I, Lux A. Zinc in plants. New Phytol. 2007;173(4):677-702.

50. Fujita M, Shinozaki K. Identification of polyamine transporters in plants: Paraquat transport provides crucial clues. Plant Cell Physiol. 2014;55:855-61.

51. Wang $H$, Wang $H$, Shao $H$, Tang $X$. Recent advances in utilizing transcription factors to improve plant abiotic stress tolerance by transgenic technology. Front Plant Sci. 2016;7:67. https://doi.org/10.3389/fpls.2016.00067.

52. Alscher RG, Erturk N, Heath LS. Role of superoxide dismutases (SODs) in controlling oxidative stress in plants. J Exp Bot. 2002;53:1331-41.

53. Küpper H, Lombi E, Zhao FJ, McGrath SP. Cellular compartmentation of cadmium and zinc in relation to other elements in the hyperaccumulator Arabidopsis halleri. Planta. 2000;212(1):75-84.

54. Zhao FJ, Lombi E, Breedon TM. Zinc hyperaccumulation and cellular distribution in Arabidopsis halleri. Plant Cell Environ. 2000;23:507-14. https:// doi.org/10.1046/j.1365-3040.2000.00569.x.
55. Becher M, Talke IN, Krall L, Krämer U. Cross species microarray transcript profiling reveals high constitutive expression of metal homeostasis genes in shoots of the zinc hyperaccumulator Arabidopsis halleri. Plant J. 2004;37(2):251-68.

56. Talke IN, Hanikenne M, Krämer U. Zinc-dependent global transcriptional control, transcriptional deregulation, and higher gene copy number for genes in metal homeostasis of the hyperaccumulator Arabidopsis halleri. Plant Physiol. 2006;142(1):148-67.

57. Mirouze M, Sels J, Richard O, Czernic P, Loubet S, Jacquier A, François IE, Cammue $B$, Lebrun M, Berthomieu P, Marquès L. A putative novel role for plant defensins: a defensin from the zinc hyper accumulating plant, Arabidopsis halleri, confers zinc tolerance. Plant J. 2006:47(3):329-42.

58. Moreno I, Norambuena L, Maturana D, Toro M, Vergara C, Orellana A, ZuritaSilva A, Ordenes VR. AtHMA1 is a thapsigargin-sensitive Ca2+/heavy metal pump. J Biol Chem. 2008;283(15):9633-41.

59. Kim YY, Choi H, Segami S, Cho HT, Martinoia E, Maeshima M, Lee Y AtHMA1 contributes to the detoxification of excess $\mathrm{Zn}(\mathrm{II})$ in Arabidopsis. Plant J. 2009;58(5):737-53. https://doi.org/10.1111/j.1365-313X.2009.03818.x.

60. Xiao S, Chye ML. New roles for acyl-CoA-binding proteins (ACBPs) in plant development, stress responses and lipid metabolism. Prog Lipid Res. 2011 50:141-51.

61. Naya L, Paul S, Valdés-López O, Mendoza-Soto AB, Nova-Franco B, SosaValencia G, Reyes JL, Hernández G. Regulation of copper homeostasis and biotic interactions by microRNA 398b in common bean. PLoS One. 2014; 9(1):e84416.

62. Xu J, Wang XY, Guo WZ. The cytochrome P450 superfamily: key players in plant development and defense. J Integra Agric. 2015;14(9):1673-86.

63. Chepyshko H, Lai CP, Huang LM, Liu JH, Shaw JF. Multifunctionality and diversity of GDSL esterase/lipase gene family in rice (Oryza sativa L. japonica) genome: new insights from bioinformatics analysis. BMC Genomics. 2012;13(1):309.

64. Fan J, Yan C, Roston R, Shanklin J, Xu C. Arabidopsis lipins, PDAT1 acyltransferase, and SDP1 triacylglycerol lipase synergistically direct fatty acids toward $\beta$-oxidation, thereby maintaining membrane lipid homeostasis. Plant Cell. 2014;26:4119-34

65. Fan J, Yu L, Xu C. A central role for triacylglycerol in membrane lipid breakdown, fatty acid $\beta$-oxidation, and plant survival under extended darkness. Plant Physio. 2017;174(3):1517-30. https://doi.org/10.1104/pp.17.00653.

66. Fan J, Yan C, Xu C. Phospholipid:diacylglycerol acyltransferase-mediated triacylglycerol biosynthesis is crucial for protection against fatty acid-induced cell death in growing tissues of Arabidopsis. Plant J. 2013;76(6):930-42.

67. Oteiza PI, Clegg MS, Zago MP, Keen CL. Zinc deficiency induces oxidative stress and AP-1 activation in 3T3 cells. Free Radic Biol Med 2000:28(7):1091-9.

\section{Submit your next manuscript to BioMed Central and we will help you at every step:}

- We accept pre-submission inquiries

- Our selector tool helps you to find the most relevant journal

- We provide round the clock customer support

- Convenient online submission

- Thorough peer review

- Inclusion in PubMed and all major indexing services

- Maximum visibility for your research

Submit your manuscript at www.biomedcentral.com/submit
) Biomed Central 